\section{Searching for a placental derived ES-62-like molecule to explain rheumatoid arthritis amelioration in pregnancy}

\author{
Craig D. Scoville, ${ }^{1}$ Devon Rasmussen² \\ 1'nstitute of Arthritis Research, Idaho \\ Falls, ID, 2Department of Biology, Idaho \\ State University, Pocatello, ID, USA
}

\section{Abstract}

The majority of women with rheumatoid arthritis (RA) experience disease amelioration during pregnancy for unclear reasons. One possible explanation pursued and described here is whether the placenta produces a protein similar to the immunomodulating protein, ES-62, excreted by filarial nematodes. This protein has also been shown to reduce disease activity in animal models of RA. Eleven human placentas were prepared and a polyclonal antiES-62 antiserum was used to identify if any ES-62- like molecule exists from human placental tissues. Any bands identified were then excised from the gel and sent for mass spectrometry and protein identification. The antiserum showed consistent cross reactivity with the heavy chain from immunoglobulin G (IgG) from the eleven human placentas by mass spectrometry. No primary sequence homology between the heavy chain of IgG and ES-62 was identified. The placenta does not produce an ES-62-like molecule. However the binding of the antiserum to the Fc region of IgG suggests that this may be a possible mechanism for rheumatoid factor production in some patients with chronic filarial infections.

\section{Introduction}

About $70-80 \%$ of pregnant women with rheumatoid arthritis (RA) may experience disease amelioration during pregnancy. ${ }^{1-5}$ However the mechanism of action by which this phenomenon occurs is unclear. ${ }^{6}$ The Th1/Th2 hypothesis by Wegmann proposed that normal pregnancy induced a bias toward Th2 cytokine production. ${ }^{7}$ Such a cytokine bias would reduce RA activity since RA is predominantly a Thl driven disease. ${ }^{8}$ This hypothesis has been challenged with a broader involvement of both NK cells and T cells producing predominantly type 2 rather than type 1 cytokines in normal pregnancy.9,10 The mechanism by which this cytokine bias occurs is unclear.
A possible hypothesis for the amelioration of pregnant women with rheumatoid arthritis is to consider that the placenta is serving in a parasitic role and modulating the immune system in the same way that parasitic infections dampen the host's immune response. Parasitic infections typically exemplify long term survival by down regulating the inflammatory response by the host, which is to the advantage of the parasite and also the host. ${ }^{11}$ Therefore, could the placenta be producing a parasitic-like protein that is dampening the immune response to the fetus and also be dampening the immune response to the host's joints? of interest is the fact that a filarial derived protein, known as ES-62, has immunomodulating properties and has been shown to reduce disease activity in animal models of RA. ${ }^{12-16}$ This protein has also been shown to induce a Th2 response. ${ }^{17}$ If the placenta serves a parasitic role in enabling the fetus to survive, one may ask if the placenta produces an ES-62- like molecule which modulates the immune system during normal pregnancy to cause a preferential type 2 over type 1 cytokine bias and thereby explain disease amelioration in RA. The ES-62 molecule is a $62 \mathrm{kDa}$ glycoprotein secreted as a tetramer by the rodent filarial nematode Acanthocheilonema viteae and also produced by other filarial nematodes. ${ }^{12}$ It appears that the primary anti-inflammatory mechanism of action of ES-62 is mediated by the phosphorylcholine moieties expressed by this glycoprotein. ${ }^{16}$ Other work has shown that placental post-translational modification with phosphocholine of secretory polypeptides occurs and may play a role in the immunomodulation that takes place during pregnancy. ${ }^{18}$

The focus of this research project sought to answer the question of whether human placentas produce an ES-62- like molecule. In order to maximize the possibility of finding such a molecule, a polyclonal anti-ES-62 antiserum (generously provided by Dr William Harnett) was used which could detect different features of the ES-62 molecule and thereby enlarge the tracking field for finding an ES-62like molecule. ${ }^{19}$ The strategy employed in this pursuit consisted of using this polyclonal antibody to identify on Western Immunoblotting any placental derived proteins that did react with the polyclonal antiserum and then subject such protein bands to mass spectrometry for comparison with the ES-62 molecule.

\section{Materials and Methods}

\section{Placental preparation}

Eleven placentas were obtained from pregnant women who delivered healthy babies and consented using IRB approved informed con-
Correspondence: Craig Davis Scoville, Institute of Arthritis Research, 2220 East 25th Street, Idaho Falls, ID 83404, USA.

Tel.: +1.208.542.9080 - Fax: +1.208.542.9081.

E-mail: cdscoville@yahoo.com

Key words: rheumatoid arthritis, pregnancy, rheumatoid factor.

Acknowledgements: the authors would thank Dr. Gene Scalarone, Professor of Biology, ISU, for his kindness and support of this project, and Roxanne Scoville for her gifted editorial skills in refining this publication, and again, Dr. William Harnett, for his generosity in providing the polyclonal anti-ES-62 anti-serum, the purified ES-62, and valuable comments.

Contributions: the authors contributed equally.

Conflict of interests: the authors declare no potential conflict of interests.

Funding: this project was funded by the Institute of Arthritis Research for which Dr. Scoville is the presiding officer and principal investigator.

Received for publication: 2 September 2014. Revision received: 6 November 2014

Accepted for publication: 9 November 2014.

This work is licensed under a Creative Commons Attribution NonCommercial 3.0 License (CC BYNC 3.0).

CC Copyright C.D. Scoville and D. Rasmussen, 2014 Licensee PAGEPress, Italy

Rheumatology Reports 2014; 6:5622

doi:10.4081/rr.2014.5622

sent forms to procure their respective placentas for research purposes. Of these eleven subjects, eight were healthy, two (MM and KS) had rheumatoid arthritis, and one (BV) had gestational diabetes. All of the subjects delivered at term except for BV who delivered at 35.2 weeks. Each intact placenta, once obtained, was placed in a plastic container on ice and refrigerated and within 24-48 hours the placenta was processed by removing the umbilical cord and embryonic sac. It was then washed generously in physiologic saline three times, and then homogenized with a blender at medium speed for $30 \mathrm{sec}$ and then slow speed for $1 \mathrm{~min}$. Samples were then placed into conical tubes and centrifuged at $1400 \times \mathrm{g}$ for 10 min, then the supernatant removed and stored frozen.

The tissue component was prepared by adding $10 \mathrm{~mL}$ of nanopure water to each individual conical tube on ice, then sonicating the placental tissue for $30 \mathrm{sec}$, then cool for $3 \mathrm{~min}$, then repeating this process three times for each conical tube. Then 50 cc of nanopure 
water was added and mixed to each conical tube to create a hypotonic solution and cause cell lysis. Each sample was made 1:10,000 merthiolate (v/v), incubated at 4 degrees $\mathrm{C}$ for 48 hours, centrifuged at $1400 \times \mathrm{g}$ for $25 \mathrm{~min}$, after which the lysate was removed from the conical tube and stored at $-20^{\circ} \mathrm{C}$.

A portion of mid thickness uterine tissue was obtained with appropriate informed consent from a woman having a hysterectomy for a benign indication. This tissue was processed by grinding the tissue with a mortar and pestle, suspending the sample in nanopure water, and then allowed to incubate at $4^{\circ} \mathrm{C}$ for 48 hours, and centrifuged at $1400 \times \mathrm{g}$ for $25 \mathrm{~min}$. The lysate was then removed from the conical tube and stored at $-20^{\circ} \mathrm{C}$.

\section{SDS-PAGE and Western immunoblotting}

Frozen lysate from a given placental or uterine sample, as described above, was thawed, centrifuged $10,000 \mathrm{~g} \times 13 \mathrm{~min}$, the supernatant collected, made $30 \mathrm{mM}$ EDTA (Sigma), mixed well and incubated at room temperature $\times 30$ min with gentle rocking. This solution was centrifuged at $10,000 \mathrm{~g} \times 13 \mathrm{~min}$, the supernatant collected, and treated with DNase/RNase preparation (Sigma; final concentration $5 \mathrm{ug} / \mathrm{mL}$ ), incubated at $37^{\circ} \mathrm{C}$ for 1 hour, then centrifuged $5000 \mathrm{~g} \times 10 \mathrm{~min}$. The supernatant was collected and filtered with 0.22 micron Millipore and stored at $-20^{\circ} \mathrm{C}$ and represented a purified lysate sample.

In preparation for SDS-PAGE and Western Immunoblotting, the purified lysate samples were thawed, made 1:5 HES buffer $(10 \mathrm{mM}$ HEPES, $10 \mathrm{mM}$ EDTA, $250 \mathrm{mM}$ sucrose), then added either reducing or non-reducing lane marker solution (Thermo Scientific Pierce, Waltham, MA, USA). Samples were then boiled for $10 \mathrm{~min}$, and aliquots layered onto $8 \%$ or $10 \%$ precast Precise Tris-HEPES gels (Thermo Scientific Pierce) for SDS-PAGE. After completing the electrophoresis, gels were either stained with Imperial Protein Stain (Thermo Scientific Pierce), or performed Western Immunoblotting using reagents and colorimetric testing with horseradish peroxidase (KPL, Gaithersburg, MD). When performing the Western Immunoblot the primary antibody, consisting of the rabbit polyclonal anti-ES-62 antiserum, was made 1:1000 dilution. This antiserum was provided by Dr. William Harnett, Department of Immunology, University of Strathclyde, Glasgow, UK. Details regarding this antiserum have been previously reported..$^{19}$ Briefly, Dr Harnett indicated that this antiserum was raised by injecting purified ES-62 into rabbits, and then treating the serum to Protein A column chromatography and the resulting product was shown to react with phosphorylcholine attached to N-type glycans, and also to both sequential (primary structure) and conformational (tertiary structure) epitopes. Also the polyclonal antiserum is able to recognize PC conjugated to N-type glycans but not PC conjugated to BSA. There was no non-specific binding of this antiserum to the Fc region of immunoglobulins (personal communications with Dr Harnett). Also Dr Harnett kindly provided the purified ES-62 molecule at $250 \mathrm{ug} / \mathrm{mL}$ endotoxin free, sterile, and filtered.

SDS-PAGE and Western Immunoblotting was performed on the serum samples of healthy males and non-pregnant females under reducing conditions using the polyclonal anti-ES-62 antiserum. Serum samples were obtained with appropriate informed consent.

\section{Protein identification}

An affinity chromatography column was prepared using HiTrap NHS-activated HP (GE Healthcare 17-0716-01) and followed protocol (GE Healthcare 71-7006-00) using the rabbit polyclonal anti-ES-62 antiserum. The purified placental lysate sample (KS) was subjected to affinity chromatography. The eluted sample was tested using SDS-PAGE and Western Immunoblotting under reducing conditions and revealed a $50 \mathrm{kDa}$ protein band. This same band was visualized using SDS-PAGE, stained with Imperial Protein Stain, and the band carefully excised from the gel and sent to ProtTech Inc (Norristown, PA, USA) for protein sequencing and identification using proprietary technique and mass spectrometry. In brief ProtTech's proprietary technique consists of taking the protein gel band, distaining it, cleaning it, and digesting in-gel with sequencing grade modified trypsin. The resulting peptide mixture was analyzed by a LC-MS/MS system, in which a high pressure liquid chromatography (HPLC) with a 75 micrometer inner diameter reverse phase $\mathrm{C} 18$ column has on-line coupling with an ion trap mass spectrometer. The mass spectrometric data acquired was matched to the most recent nonredundant protein database from NCBI's GenBank with ProtTech's proprietary software.

\section{Size exclusion and protein A chro- matography}

Frozen purified placental lysates from four placental tissues (KS, MP, MC, RA) were thawed, and $5 \mathrm{~mL}$ samples were each applied to a HiPrep 26/60 Sephacryl S-200 HR column at flow rate $1.0 \mathrm{~mL} / \mathrm{min}$ in $0.05 \mathrm{M}$ phosphate, $0.15 \mathrm{M} \mathrm{NaCl}$ buffered saline, $\mathrm{pH} 7.8$, and the fractions were tested for reactivity with polyclonal rabbit anti-ES-62 serum using Western Blots. The fractions from 132 to 150 minutes showed positive reactivity and therefore were pooled, and then concentrated using Millipore spin concentrators (nominal molecular weight limit $100 \mathrm{kDa}$ ), and then mixed 1:1 with
Protein A IgG Binding buffer $(0.02 \quad \mathrm{M}$ NaCitrate, 0.1M phosphate buffer, $\mathrm{pH} 7.4$, Thermo Scientific Pierce), and then applied to Protein A column (5 mL Protein A/column, Thermo Scientific Pierce). The column was washed with binding buffer at $10 \times$ column volume and until the absorption was zero, and then eluted immunoglobulin with Immunopure IgG elution buffer $(0.02 \mathrm{M}$ NaCitrate, $0.1 \mathrm{M} \mathrm{NaCl}, \mathrm{pH} 2.5$, Thermo Scientific Pierce) at $1 \mathrm{~mL} / \mathrm{min}$ and collected fractions. These fractions were made $0.05 \mathrm{M}$ phosphate, $0.15 \mathrm{M} \mathrm{NaCl}$ buffered saline, pH 7.4 using a desalting column and then the samples were concentrated using Millipore concentrators by performing about 2-3 cycles of concentrating and then adding buffer. Protein concentrations were assayed using BCA technique and then samples stored at -20 degrees centigrade. When ready for testing, a given sample was thawed and then subjected to SDS-PAGE.

\section{Protein A purification and papain digest of immunoglobulin $\mathrm{G}$}

The serum of subject TR was made 1:1 with Protein A IgG Binding buffer $(0.02 \mathrm{M}$ NaCitrate, 0.1M phosphate buffer, pH 7.4, Thermo Scientific Pierce), applied to Protein A column as described above. The final eluted and concentrated product was then treated with immobilized papain (Thermo Scientific Pierce), and then the fractions passed over a Protein A column again and the non-eluted and eluted fractions tested by SDS-PAGE and Western Immunoblotting.

\section{Results}

\section{Does an ES-62- like molecule exist in the human placenta?}

The polyclonal antibody to ES-62 was shown to bind cleanly to the ES-62 molecule, thereby serving as a positive control (Figure 1D). Then the purified placental tissue lysate from 11 donors were prepared using HES buffer and examined under non-reducing conditions using SDS-PAGE and Western Immunoblotting and showed the characteristic pattern of immunoglobulin $\mathbf{G}$ intracellular assembly (Figure 1A) consisting of H2L2, H2L, H2, and H chains (H: heavy chain; L: light chain). The same samples were subsequently tested under reducing conditions using SDS-PAGE and Western Immunoblotting and showed predominantly the $\mathrm{H}$ chain band (Figure 1B). These findings suggested that the polyclonal anti-ES62 anti-serum was not identifying the characteristic pattern of ES-62 on Western Immunoblotting but rather recognizing the features of an immunoglobulin $\mathrm{H}$ chain. 


\section{Characterization of the placental derived immunoglobulin $\mathrm{G}$}

As described in Materials and Methods, an aliquot of the purified placental lysate of KS was poured over an affinity column made with the rabbit polyclonal anti-ES-62 antiserum. The eluted sample was subjected to SDS-PAGE under reducing conditions, and the Western Immunoblotting revealed a $50 \mathrm{kDa}$ molecular weight protein similar to what is shown in slot 2 on Figure 1B. The eluted sample was again tested with SDS-PAGE only and the gel stained with Imperial Protein Stain. This protein band, corresponding to the $50 \mathrm{kDa}$ protein, was excised and sent for protein analysis. These findings (Table 1) showed only one protein or hit that corresponded to the eleven different polypeptide sequences identified by mass spectrometry. The protein identified by mass spectrometry had a protein mass of 52,766.1 $\mathrm{kDa}$ and was identified as being a match to IgG1 H chain with BLAST identification number AAH69016.1. There was no evidence of any sequences corresponding to ES-62 molecule.

In order to validate this finding, four purified placental lysates (KS, MP, MC, RA) were all subjected to size exclusion chromatography. The fractions were tested using SDS-PAGE and Western Immunoblotting using the polyclonal anti-ES-62 antiserum and the fractions shown in Figure 2 had positive reactivity with the antiserum.

These fractions were collected, concentrated, and tested with Protein A column chromatography. The eluted samples were tested under reducing conditions with SDS-PAGE and the identified $50 \mathrm{kDa}$ molecular weight band sent for protein analysis using mass spectrometry. Table 2 shows that the antiserum does indeed cross react with primarily the $\mathrm{H}$ chain of IgG. No ES-62- like molecular sequences were identified. The mass spectrometry did not report on carbohydrate side chains or the presence of phosphocholine. Of note, the PC found in ES-62 is conjugated to N-type glycans - and PC-glycans are not present in humans. ${ }^{12}$ To determine if these findings were unique to placental samples, the serum of healthy males and non pregnant females were tested with the polyclonal antiserum. This testing revealed that the polyclonal antiserum was binding to all IgG $\mathrm{H}$ chains found in the sampled subjects and there was no reactivity with alpha-fetoprotein (Figure 1C). There was reactivity with uterine tissue lysate suggesting infiltration of IgG into uterine tissue or cross reactivity with carbohydrate side chains on glycoproteins. As the rabbit polyclonal anti-ES-62 anti-serum shows no binding to the presumed L chain, it's important to identify whether the antiserum was seeing the Fab or Fc portion of the $\mathrm{H}$ chain. Therefore protein A purified IgG from the serum of TR was treated with papain and then subjected to SDS-PAGE and Western

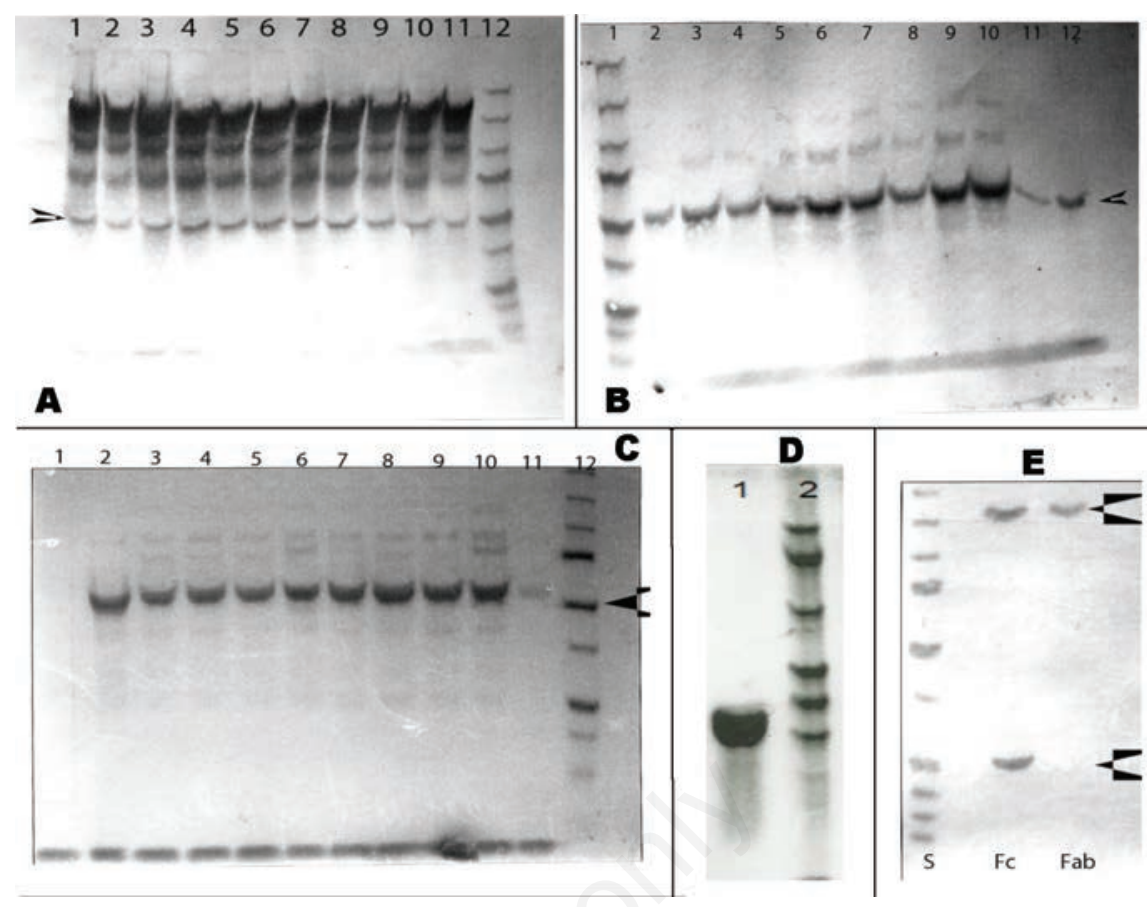

Figure 1. A) SDS-PAGE under non-reducing conditions and Western Immunoblot of purified placental lysates from eleven donors (1:MM, 2:MP, 3:KP, 4:CB, 5:MC, 6:CH, 7:SB, 8:JC, 9:BV, 10:BC, 11:KS) using the rabbit polyclonal anti-ES-62 antiserum, and slot 12 represents the BioRad Precision Plus Protein Standards consisting of molecular weights $250 \mathrm{k}, 150 \mathrm{k}, 100 \mathrm{k}, 75 \mathrm{k}, 50 \mathrm{k}, 37 \mathrm{k}, 25 \mathrm{k}, 20 \mathrm{k}, 15 \mathrm{k}$, and $10 \mathrm{k}$. The arrow corresponds to the $50 \mathrm{k}$ protein marker. B) SDS-PAGE under reducing conditions and Western Immunoblot of purified placental lysates from eleven donors as listed below $(2: \mathrm{KS}, 3: \mathrm{BC}$, 4:BV, 5:JC, 6:SB, 7:CH, 8:MC, 9:CB, 10:KP, 11:MP, 12:MM) using the rabbit polyclonal anti-ES-62 antiserum. Again the arrow marks the 50k protein marker. C) SDS-PAGE under reducing conditions and Western Immunoblotting using the rabbit polyclonal anti-ES-62 antiserum: Column 1: alpha-fetoprotein, 2: RR (uterine tissue), 3: AMH, 4: LMG, 5: HDZ, 6: RND, 7: JRF, 8: BSD, 9: BRH, 10: EML, 11: BC, 12: standard. Serum samples of healthy non pregnant women are represented in columns 3 thru 6 , serum samples of healthy adult males are represented in columns 7 thru 10. The protein A purified placental derived IgG from BC is represented in column 11. D) Western Immunoblot of the SDS-PAGE (8\% gel) of purified ES-62 (slot 1) using the rabbit polyclonal anti-ES-62 antiserum. Slot 2 is the GE HealthCare Amersham high molecular weight standards of $220 \mathrm{k}, 170 \mathrm{k}, 116 \mathrm{k}, 76 \mathrm{k}, 70 \mathrm{k}$, and 53k. E) SDS-PAGE under non-reducing conditions and Western Immunoblotting (using the rabbit polyclonal anti-ES-62 antiserum) of a protein A purified sample of TR serum, treated with papain, and then passed over a protein $A$ column for which the fractions not binding to the column are represented by Fab. The fractions binding to the column were then eluted and are represented by Fc. The upper arrow represents the intact IgG that was not completely digested by the papain and the lower arrow corresponds to the $50 \mathrm{k}$ protein marker. The " $S$ " column represents the protein markers.

Table 1. Mass spectrometry of trypsin digested $50 \mathrm{kDa}$ protein of KS (1 hit; 11 peptides identified; sequence header: IgG1 H; chain AAH69016).

\begin{tabular}{llc}
\hline Protein mass & Sequence & Sequence numbers \\
1149.62 & GTLVTVSSASTK & $134-145$ \\
837.50 & ALPAPIEK & $351-358$ \\
\hline 1185.64 & GPSVFPLAPSSK & $146-157$ \\
1676.79 & FNWYVDGVEVHNAK & $299-312$ \\
\hline 2543.12 & GFYPSDIAVEWESNGQPENNYK & $395-416$ \\
1872.91 & TTPPVLDSDGSFFLYSK & $417-433$ \\
\hline 1807.00 & WSVLTVLHQDWLNGK & $326-341$ \\
\hline
\end{tabular}

This sample of KS was obtained by affinity chromatography and SDS-PAGE, and the protein band excised and analyzed as described in Methods and Materials. The sequence numbers correspond to the sequences found on the reference BLAST protein. 
Immunoblotting (Figure 1E). This demonstrated that the polyclonal anti-ES-62 anti-serum is binding to the $\mathrm{Fc}$ portion of the $\mathrm{H}$ chain. Interestingly the carbohydrate moieties on immunoglobulin $\mathrm{G}$ are selectively attached to the $\mathrm{CH} 2$ domain in the $\mathrm{Fc}$ region at $\mathrm{N}$-linked glycosylation site at asparagine-297. ${ }^{20}$ To better understand the cross reactivity occurring between the polyclonal anti-ES-62 antiserum with the heavy chain of immunoglobulin G, a BLAST search was conducted. The reference immunoglobulin $\mathrm{G}$ heavy chain identified with KS (accession number AAH69016.1) was compared to the aminopeptidase ES-62 molecule (accession number AAC28365.1). This comparative search revealed no primary sequence similarity with an E-score 0.26 . Therefore any cross reactivity between the polyclonal antibody and immunoglobulin $\mathrm{G}$ heavy chain must be due to shared carbohydrate moieties. The only carbohydrate moieties identified in IgG proteins are localized in the Fc region - the same region shown on papain digestion to still show cross reactivity with the antiserum.

\section{Discussion and Conclusions}

The purpose of this research was to test whether the placenta produced an ES-62- like molecule, thereby providing an explanation for the ameliorating effect of pregnancy on patients with rheumatoid arthritis. As the nature of a putative placental derived ES-62 like molecule was not known at the onset of this investigation, the use of a heterogeneous polyclonal antibody to ES-62 provided a broader scope for testing. This polyclonal anti-ES-62 antiserum recognized several features of the ES-62 molecule inclusive of the phosphorylcholine conjugated to the N-type glycans, and primary and tertiary structures. The cross reactivity between the polyclonal antiserum and human placental lysates was due to the heavy chain of immunoglobulin G. Based on mass spectrometry, no primary ES-62 structure was identified.

The cross reactivity between the polyclonal antiserum and the heavy chain of immunoglobulin G must be due to similar carbohydrate moieties. The carbohydrate structures of ES-62 and immunoglobulin G share a common core of sugars consisting of $\mathrm{N}$-acetylglucosamine conjugated to mannose, and then mannose linked to $\mathrm{N}$-acetylglucosamine. ${ }^{12,20}$ Whereas the phosphocholine in ES-62 is conjugated to $\mathrm{N}$-type glycans, no such conjugation has been shown to exist in humans. Therefore the common antigenic structure between these glycoproteins must be the core sugars without phosphocholine. Interestingly this observation of seeing the polyclonal antiserum bind to immunoglobulin $\mathrm{G}$ defines the poly- clonal antibody as a rheumatoid factor and may explain the occurrence of rheumatoid factor in chronic filarial infections. Hamilton $e t$ al. did make a comment regarding their experience of $16 \%$ prevalence of rheumatoid factor in patients with filariasis. ${ }^{21}$

The original observation that pregnancy improves the signs and the symptoms of about $70-80 \%$ of pregnant women with rheumatoid arthritis (RA) was reported by Phillip Hench in

Table 2. Mass Spectrometry of the $50 \mathrm{kDa}$ proteins of modified placental lysates.

\begin{tabular}{|c|c|c|}
\hline Placental donor, hits & N. of peptides identified & Sequence header \\
\hline $\begin{array}{r}\mathrm{KS} \\
1 \\
2 \\
3 \\
4 \\
5 \\
6\end{array}$ & $\begin{array}{l}67 \\
15 \\
5 \\
2 \\
1 \\
1\end{array}$ & $\begin{array}{l}\text { IgG1 H chain } \\
\text { Ig kappa light chain VLLJ region } \\
\text { Ig heavy chain variable region } \\
\text { Ig heavy chain variable region } \\
\text { Ig heavy chain variable region } \\
\text { Ig kappa light chain V region }\end{array}$ \\
\hline $\begin{array}{r}\text { BC } \\
1 \\
2 \\
3 \\
4 \\
5 \\
6 \\
7 \\
8\end{array}$ & $\begin{array}{l}58 \\
4 \\
3 \\
1 \\
1 \\
1 \\
1 \\
1\end{array}$ & $\begin{array}{l}\text { IgG H chain } \\
\text { Anti-tetanus toxin single chain Fv } \\
\text { Ig H chain variable region } \\
\text { IgM H chain variable region } \\
\text { Ig H chain V-III region } \\
\text { Anti-tetanus toxoid H chain variable } \\
\text { Ig H chain variable region } \\
\text { Ig M rearranged H chain mRNA V-D-J }\end{array}$ \\
\hline $\begin{array}{r}\text { MP } \\
1 \\
2 \\
3 \\
4 \\
5 \\
6\end{array}$ & $\begin{array}{l}28 \\
2 \\
2 \\
1 \\
1 \\
1\end{array}$ & $\begin{array}{l}\text { IgG H chain } \\
\text { Mouse Ig H chain V region } \\
\text { Ig H chain VHDJ region } \\
\text { Ig H chain VHDJ region } \\
\text { Ig H chain variable region } \\
\text { Ig H chain variable region }\end{array}$ \\
\hline $\begin{array}{r}\text { RA } \\
1 \\
2 \\
3 \\
4 \\
5 \\
\end{array}$ & $\begin{array}{l}121 \\
17 \\
5 \\
2 \\
2\end{array}$ & $\begin{array}{l}\text { IgG1 H chain } \\
\text { Ig kappa light chain VLJ region } \\
\text { Ig H chain variable region } \\
\text { Ig H chain variable region } \\
\text { Ig kappa chain } \mathrm{V} \text { region }\end{array}$ \\
\hline
\end{tabular}

The purified placental lysates from 4 donors were prepared by size exclusion chromatography and then protein A chromatography and then the $50 \mathrm{kDa}$ protein band excised and sent for protein analysis using mass spectrometry. The proteins identified using this technique are designated by hits and listed in order of the frequency of that protein. Some peptides identified represent polypeptide repeats. The sequence header represents each protein matching the BLAST search.

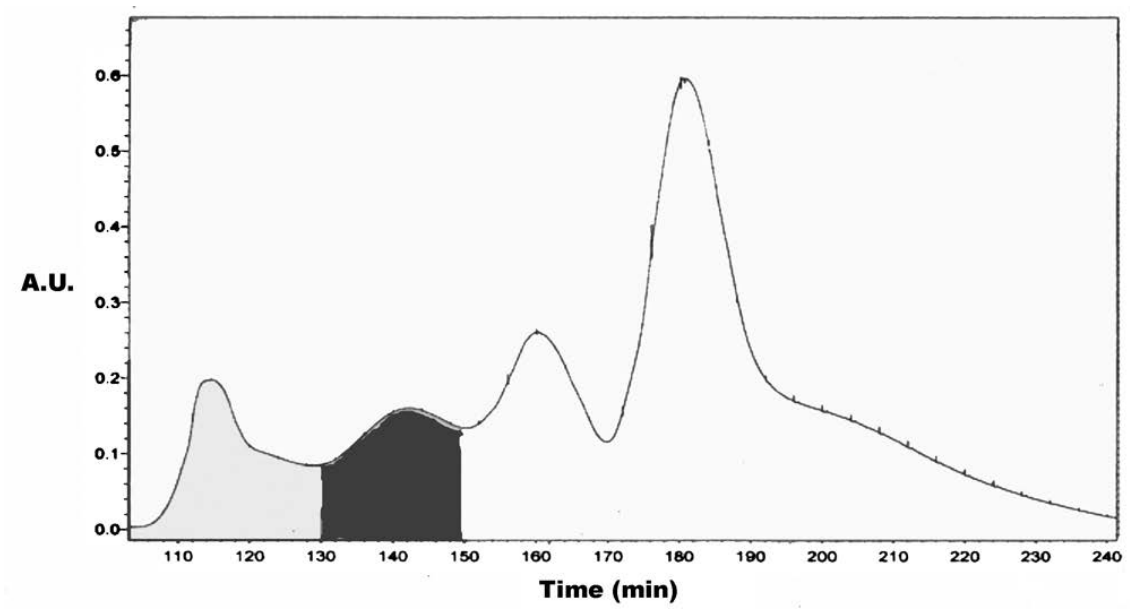

Figure 2. Sephadex S-200 size exclusion chromatography of the prepared purified placental lysate of $\mathrm{KS}, \mathrm{BC}, \mathrm{MP}$, and $\mathrm{RA}$ and the darkened area under the curve represents the fractions collected and tested by SDS-PAGE and Western Immunoblotting and found to cross react with the anti-serum to ES-62. 
$1938^{, 22}$ and was believed to be due to some $s u b$ stance $X^{6,23}$ This observation contributed eventually to the discovery of corticosteroids and for which the Nobel Prize in Medicine in 1950 was awarded to Drs. Edward Kendall and Phillip Hench and Tadeus Reichstein. There have been several other reports confirming that the majority of women with RA do experience disease amelioration during pregnancy. ${ }^{6}$ One report demonstrated the ameliorating effect of a postpartum autologous plasma transfusion in a patient with rheumatoid arthritis. ${ }^{24}$ Dr Hench believed that corticosteroids were substance $X,{ }^{25}$ but subsequent studies have not supported this claim. ${ }^{6}$ One hypothesis tested in this work was whether an ES-62- like molecule existed that might represent substance $X$. In pursuit of substance $X$ this work showed that the placenta does not produce an ES-62 like protein. This study did not rule out possible post translational modification of the immunoglobulin $\mathrm{H}$ chain directly with phosphocholine per the work by Lovell $e t$ $a l{ }^{18}$ But to date no such modifications of immunoglobulins have been reported. ${ }^{26,27}$

\section{References}

1. Oka M. Effect of pregnancy on the onset and course of rheumatoid arthritis. Ann Rheum Dis 1953;12:227-9.

2. Neely NT, Persellin RH. Activity of rheumatoid arthritis during pregnancy. Tex Med 1977;73:59-63.

3. Ostensen M, Aune B, Husby G. Effect of pregnancy and hormonal changes on the activity of rheumatoid arthritis. Scand J Rheumatol 1983;12:69-72.

4. Barrett JH, Brennan P, Fiddler M, Silman AJ. Does rheumatoid arthritis remit during pregnancy and relapse postpartum? Results from a nationwide study in the United Kingdom performed prospectively from late pregnancy. Arthritis Rheum 1999;42:1219-27.

5. Nelson JL, Ostensen M. Pregnancy and rheumatoid arthritis. Rheum Dis Clin North Am 1997;23:195-212.

6. Golding A, Haque UJ, Giles JT. Rheumatoid arthritis and reproduction. Rheum Dis Clin North Am 2007;33:319-43.

7. Wegmann TG, Lin H, Guilbert L, Mosmann TR. Bidirectional cytokine interactions in the maternal-fetal relationship: is successful pregnancy a TH2 phenomenon? Immunol Today 1993;14:353-56

8. Gayed M, Gordon C. Pregnancy and rheumatic diseases. Rheumatol 2007;46:1634-40.

9. Sargent IL, Borzychowski AM, Redman CWG. NK cells and human pregnancy: an inflammatory view. Trends Immunol 2006;27:399-404.

10. Borzychowski AM, Croy BA, Chan WL, et al. Changes in systemic type 1 and type 2 immunity in normal pregnancy and preeclampsia may be mediated by natural killer cells. Eur J Immunol 2005;35:305463.

11. Rajamanickam A, Babu S. Immunomodulation by filarial parasites. Int Trends Immunol 2013;1:12-20

12. Harnett W, Harnett MM, Byron 0 . Structural/functional aspects of ES-62: a secreted immunomodulatory phosphorycholine-containing filarial nematode glycoprotein. Curr Protein Pept Sci 2003;4:5971.

13. McInnes IB, Leung BP, Harnett M, et al. A novel therapeutic approach targeting articular inflammation using the filarial nematode-derived phosphorylcholine glycoprotein ES-62. J Immunol 2003;171: 2127-33.

14. Harnett W, McInnes IB, Harnett MM. ES62 , a filarial nematode-derived immunomodulator with anti-inflammatory potential. Immunol Lett 2004;94:27-33.

15. Harnett W, Harnett MM, Leung BP, et al. The anti-inflammatory potential of the filarial nematode secreted product, ES-62. Curr Top Med Chem 2004;4:553-9.

16. Harnett MM, Kean DE, Boitelle A, et al. The phosphorycholine moiety of the filarial nematode immunomodulator ES-62 is responsible for its anti-inflammatory action in arthritis. Ann Rheum Dis 2008;67:518-23.

17. Harnett W, Harnett MM. Filarial nematode secreted product ES-62 is an anti-inflammatory agent: therapeutic potential of small molecule derivatives and ES-62 peptide mimetics. Clin Exp Pharmacol Physiol 2006;33:511-8.

18. Lovell TM, Woods RJ, Butlin DJ, et al. Identification of a novel mammalian posttranslational modification, phosphocholine, on placental secretory polypeptides. J Mol Endocrinol 2007;39:189-98.

19. Harnett W, Worms MJ, Kapil A, et al. Origin, kinetics of circulation and fate in vivo of the major excretory-secretory product of Acanthocheilonema viteae. Parasitol 1989;99:229-39.

20. Shade KT, Anthony RM. Antibody glycosylation and inflammation. Antibodies 2013;2:392-414.

21. Hamilton RG, Hussain R, Alexander E, Adkinson NF. Limitations of the radioimmunoprecipitation polyethylene glycol assay (RIPEGA) for detection of filarial antigens in serum. J Immunol Methods 1984;68:349-66.

22. Hench PS. The ameliorating effect of pregnancy on chronic atrophic (infectious rheumatoid) arthritis, fibrositis, and intermittent hydrarthrosis. Mayo Clin Proc 1938;13:161-80.

23. Granirer LW. Clinical response of rheumatoid arthritis to postpartum plasma. J Am Med Assoc 1951;146:995-7.

24. Scoville CD. Postpartum autologous plasma transfusion: effect on RA. Ann Rheum Dis 1992;51:1342-3.

25. Hench PS. The reversibility of certain rheumatic and non-rheumatic conditions by the use of cortisone or of the pituitary adrenocorticotropic hormone. Available from: http://www.nobelprize.org/nobel_ prizes/medicine/laureates/1950/hench-lecture.pdf

26. Cunningham BA, Pflumm MN, Rutishauser U, Edelman GM. Subgroups of amino acid sequences in the variable regions of immunoglobulin heavy chains. Proc Natl Acad Sci USA 1969;64:997-1003.

27. Edelman GM, Cunningham BA, Gall WE, et al. The covalent structure of an entire gamma G immunoglobulin molecule. Proc Natl Acad Sci USA 1969;63:78-85. 